Supporting information

Additive-Triggered Chain Transfer to a Solvent in

\title{
Coordination Polymerization
}

Zhaohe Liu,${ }^{\dagger}$, Changguang Yao, $^{\dagger}$ Chunji $\mathrm{Wu}^{\dagger}$ Zhongfu Zhao ${ }^{*, \S}$ and Dongmei Cui ${ }^{*}, \dagger, \sharp$

Figure S1. D NMR spetra of the polystyrene(up) obtained in deuterium toluene and toluene- $d 8$ (down). $\left(\mathrm{C}_{2} \mathrm{D}_{2} \mathrm{Cl}_{4}\right.$ as the internal standard, $\left.\mathrm{CHCl}_{3}, 25^{\circ} \mathrm{C}\right)$.

Figure S2. ${ }^{1} \mathrm{H}$ NMR spectrum of polystyrene $\left(M_{\mathrm{n}}=3000, M_{\mathrm{w}} / M_{\mathrm{n}}=1.4\right)$ obtained in toluene. $\left(25^{\circ} \mathrm{C}, \mathrm{CDCl}_{3}\right)$.

Figure S3. ${ }^{13} \mathrm{C}$ NMR spectrum of polystyrene $\left(M_{\mathrm{n}}=3000, M_{\mathrm{w}} / M_{\mathrm{n}}=1.4\right)$ obtained in toluene. $\left(25^{\circ} \mathrm{C}, \mathrm{CDCl}_{3}\right)$.

Figure S4. DEPT135 NMR spectrum of polystyrene $\left(M_{\mathrm{n}}=3000, M_{\mathrm{w}} / M_{\mathrm{n}}=1.4\right)$ obtained in toluene. $\left(25^{\circ} \mathrm{C}, \mathrm{CDCl}_{3}\right)$.

Figure S5. DEPT90 NMR spectrum of polystyrene $\left(M_{\mathrm{n}}=3000, M_{\mathrm{w}} / M_{\mathrm{n}}=1.4\right)$ obtained in toluene. $\left(25^{\circ} \mathrm{C}, \mathrm{CDCl}_{3}\right)$.

Figure S6. The HSQC NMR spetrum of polystyrene $\left(M_{\mathrm{n}}=3000, M_{\mathrm{w}} / M_{\mathrm{n}}=1.4\right)$ obtained in toluene. $\left(25^{\circ} \mathrm{C}, \mathrm{CDCl}_{3}\right)$.

Figure S7. The HSQC NMR spetrum of polystyrene $\left(M_{\mathrm{n}}=3000, M_{\mathrm{w}} / M_{\mathrm{n}}=1.4\right)$ obtained in toluene. (0.8 ppm-3.7ppm in ${ }^{1}$ Hand $16-53 \mathrm{ppm}$ in $\left.{ }^{13} \mathrm{C}\right)$.

Figure S8. The HSQC NMR spetrum of polystyrene $\left(M_{\mathrm{n}}=3000, M_{\mathrm{w}} / M_{\mathrm{n}}=1.4\right)$ obtained in toluene. (5.7 ppm-8.0 ppm in ${ }^{1} \mathrm{H}$ and $115-149 \mathrm{ppm}$ in $\left.{ }^{13} \mathrm{C}\right)$.

Figure S9. The HMBC NMR spetrum of polystyrene $\left(M_{\mathrm{n}}=3000, M_{\mathrm{w}} / M_{\mathrm{n}}=1.4\right)$ obtained in toluene. $\left(25^{\circ} \mathrm{C}, \mathrm{CDCl}_{3}\right)$. 
Figure S10. The HMBC NMR spetrum of polystyrene $\left(M_{\mathrm{n}}=3000, M_{\mathrm{w}} / M_{\mathrm{n}}=1.4\right)$ obtained in toluene. (0.5 ppm-3.7 ppm in ${ }^{1} \mathrm{H}$ and $15-55 \mathrm{ppm}$ in $\left.{ }^{13} \mathrm{C}\right)$.

Figure S11. The HMBC NMR spetrum of polystyrene $\left(M_{\mathrm{n}}=3000, M_{\mathrm{w}} / M_{\mathrm{n}}=1.4\right)$ obtained in toluene. (0.5 ppm-3.8 ppm in ${ }^{1} \mathrm{H}$ and $115-155 \mathrm{ppm}$ in $\left.{ }^{13} \mathrm{C}\right)$.

Figure S12. The HMBC NMR spetrum of polystyrene $\left(M_{\mathrm{n}}=3000, M_{\mathrm{w}} / M_{\mathrm{n}}=1.4\right)$ obtained in toluene. (5.7 ppm-8.0 ppm in ${ }^{1} \mathrm{H}$ and $15-55 \mathrm{ppm}$ in $\left.{ }^{13} \mathrm{C}\right)$.

Figure S13. The HMBC NMR spetrum of polystyrene $\left(M_{\mathrm{n}}=3000, M_{\mathrm{w}} / M_{\mathrm{n}}=1.4\right)$ obtained in toluene. (5.6 ppm-7.8 ppm in ${ }^{1} \mathrm{H}$ and $118-152 \mathrm{ppm}$ in ${ }^{13} \mathrm{C}$ ).

Table S1. The Effects of Various Solvent and Catalysts for Styrene Polymerization Table S2. Cartesian Coordinates for All of the Calculated Structures 


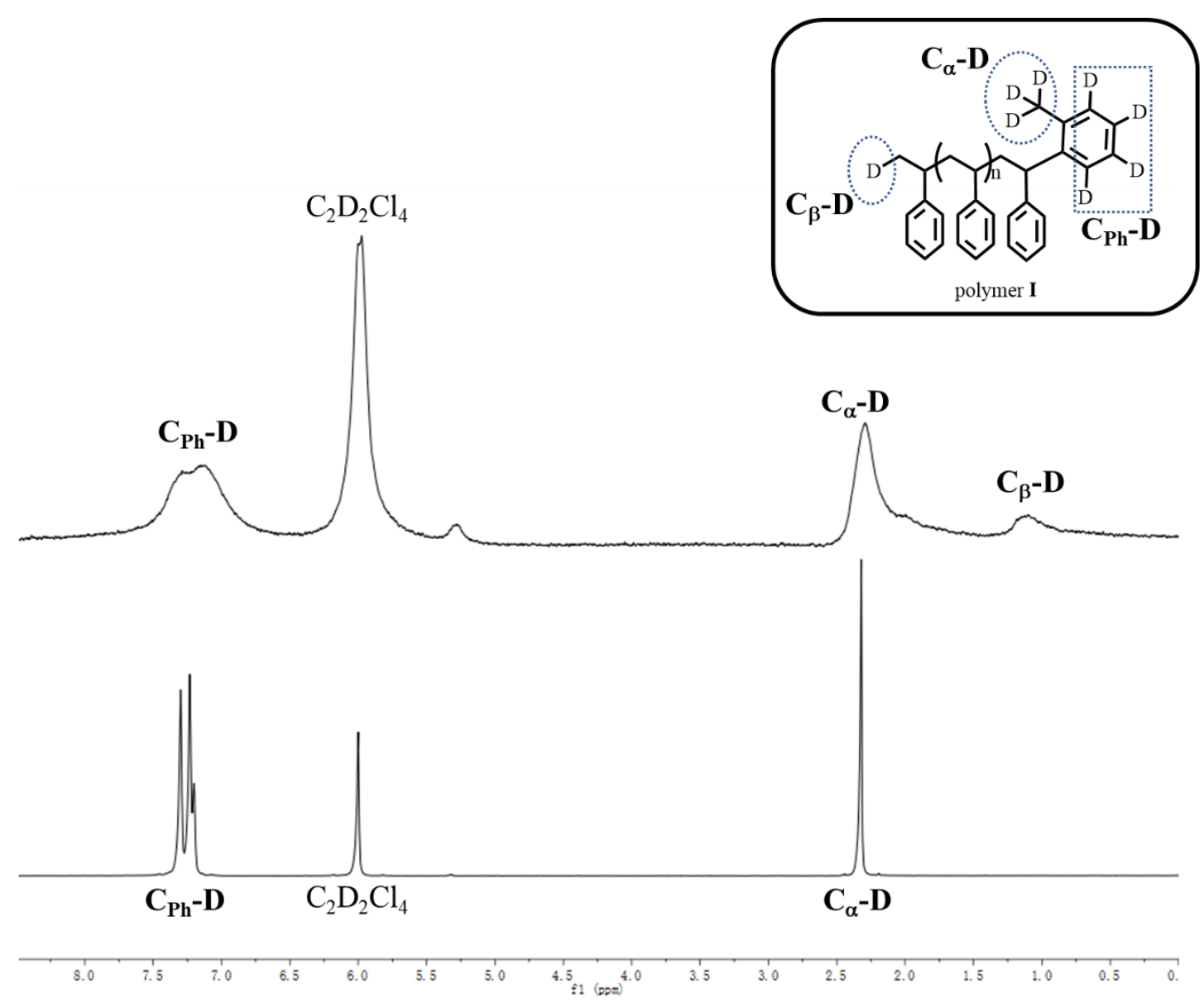

Figure S1. D NMR spetra of the polystyrene(up) obtained in deuterium toluene and toluene- $d 8$ (down). $\left(\mathrm{C}_{2} \mathrm{D}_{2} \mathrm{Cl}_{4}\right.$ as the internal standard).

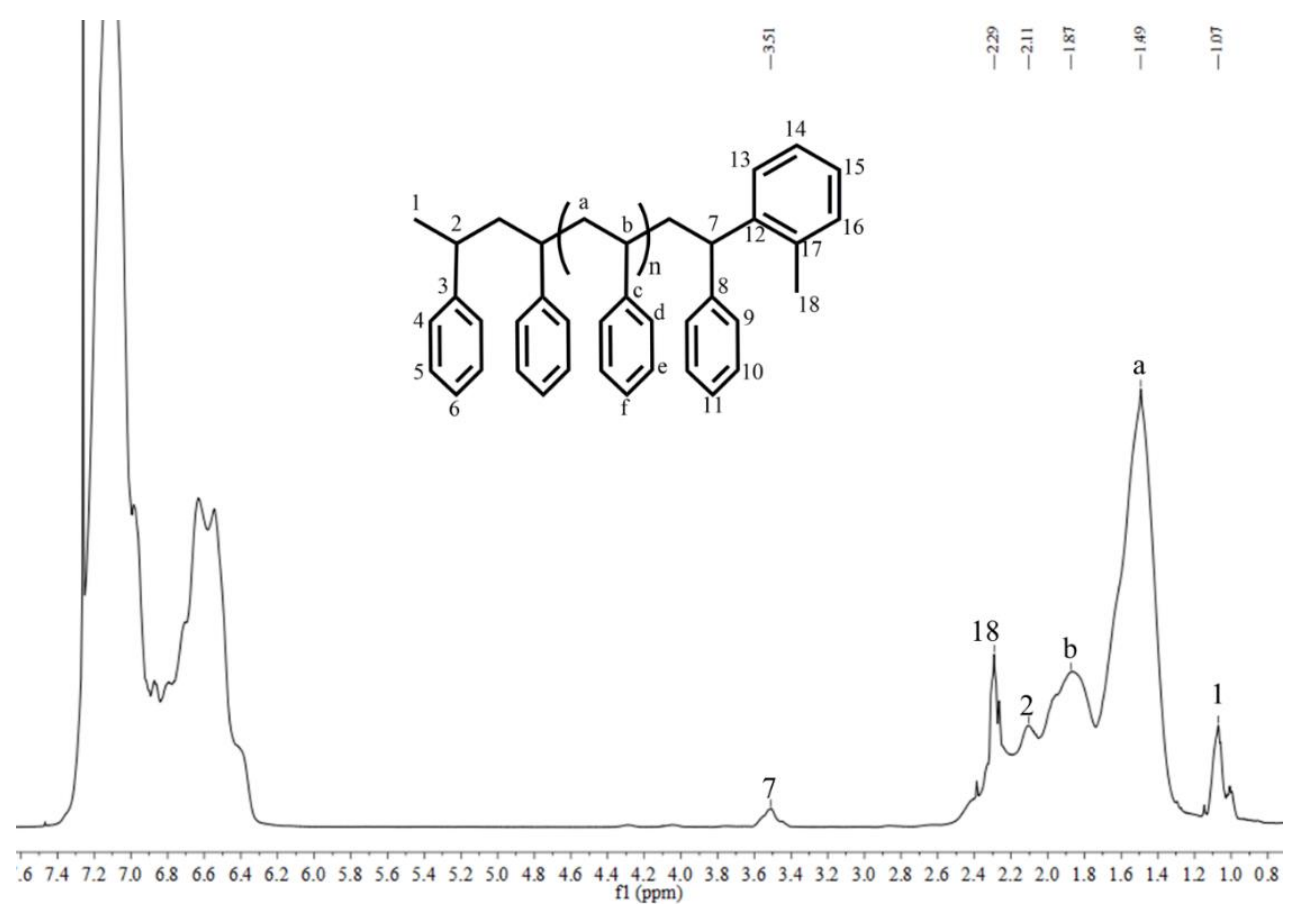

Figure S2. ${ }^{1} \mathrm{H}$ NMR spectrum of polystyrene $\left(M_{\mathrm{n}}=3000, M_{\mathrm{w}} / M_{\mathrm{n}}=1.4\right)$ obtained in toluene. $(25$ $\left.{ }^{\circ} \mathrm{C}, \mathrm{CDCl}_{3}\right)$. 


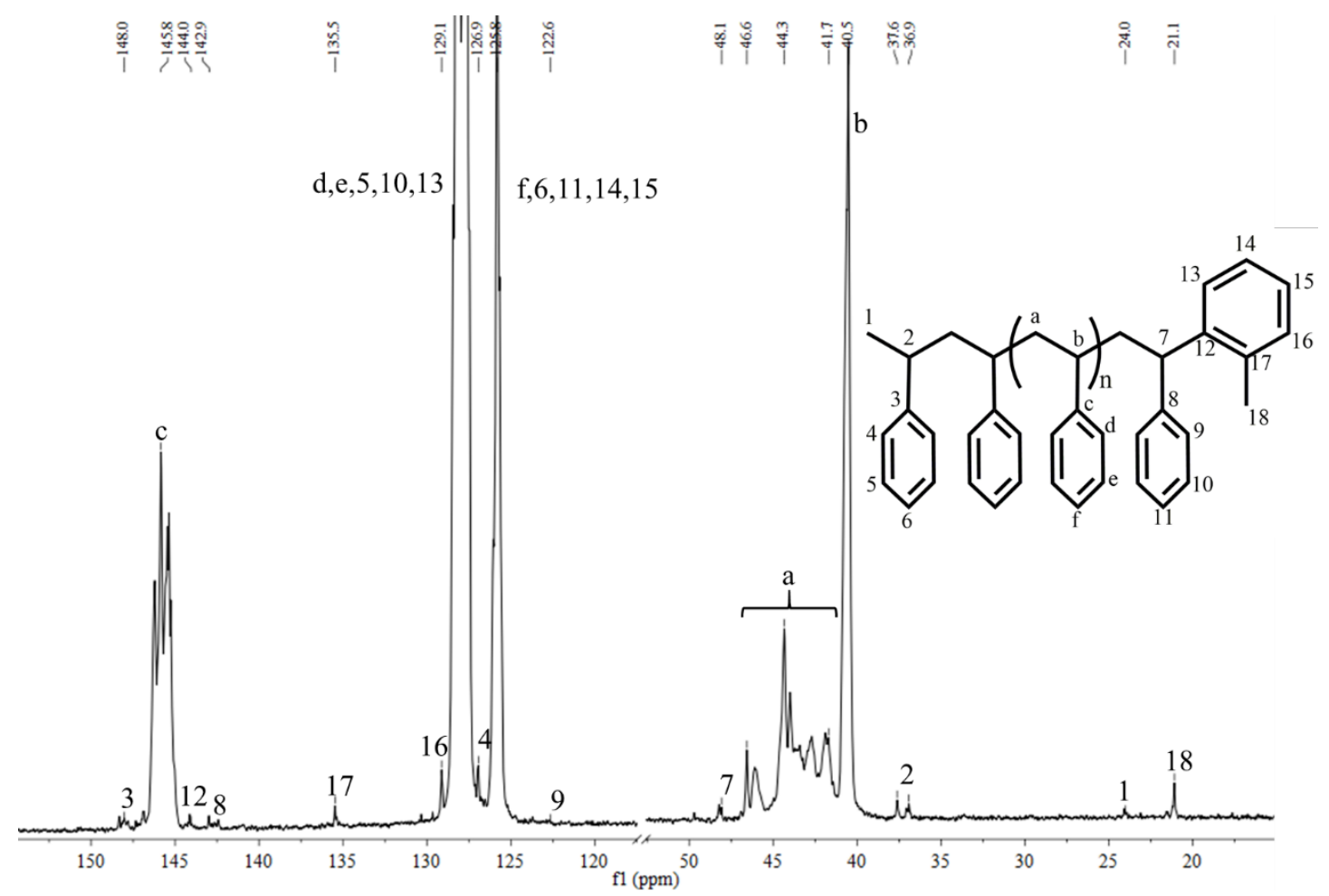

Figure S3. ${ }^{13} \mathrm{C}$ NMR spectrum of polystyrene $\left(M_{\mathrm{n}}=3000, M_{\mathrm{w}} / M_{\mathrm{n}}=1.4\right)$ obtained in toluene. (25 $\left.{ }^{\circ} \mathrm{C}, \mathrm{CDCl}_{3}\right)$.

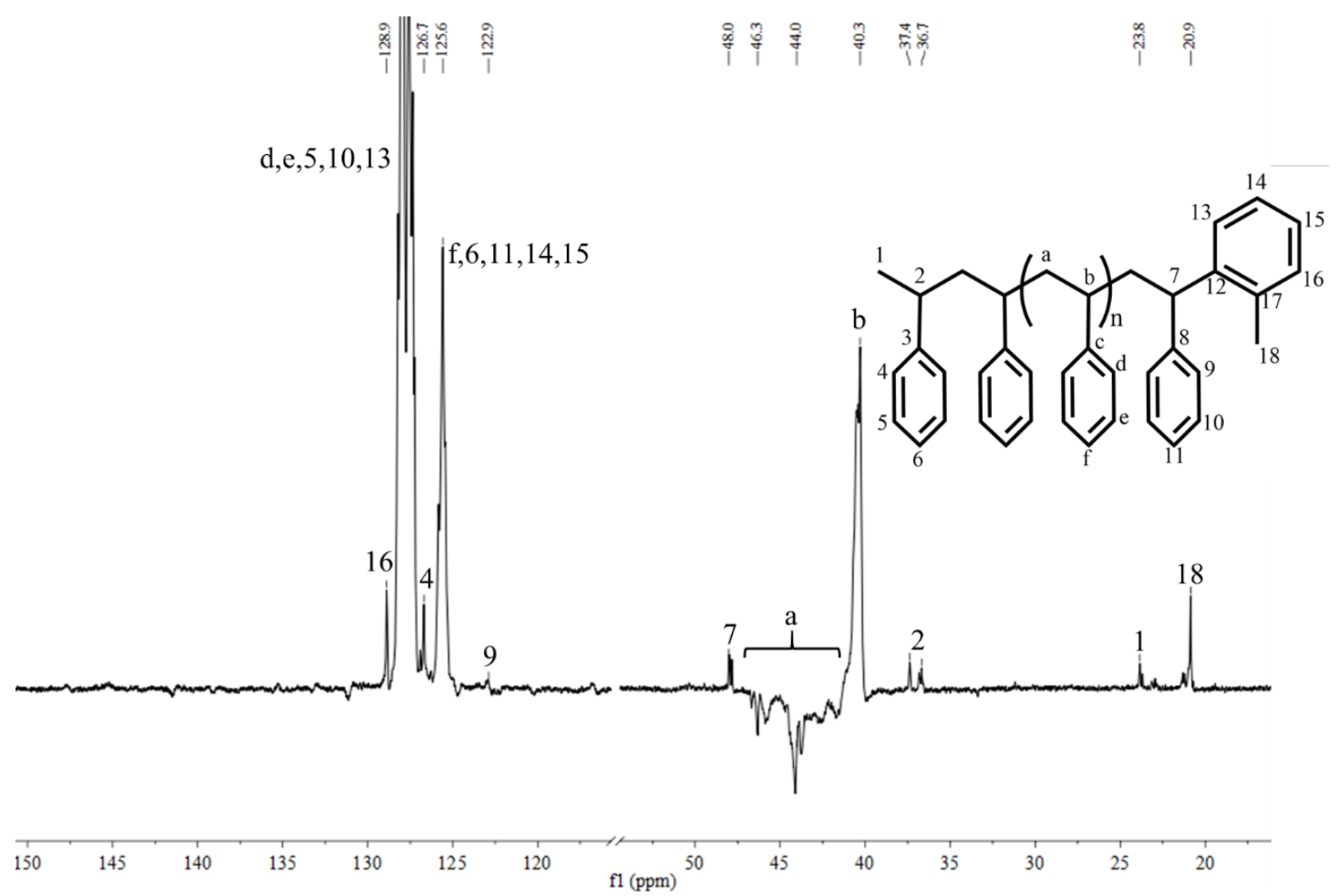

Figure S4. DEPT135 NMR spectrum of polystyrene $\left(M_{\mathrm{n}}=3000, M_{\mathrm{w}} / M_{\mathrm{n}}=1.4\right)$ obtained in toluene. $\left(25^{\circ} \mathrm{C}, \mathrm{CDCl}_{3}\right)$. 


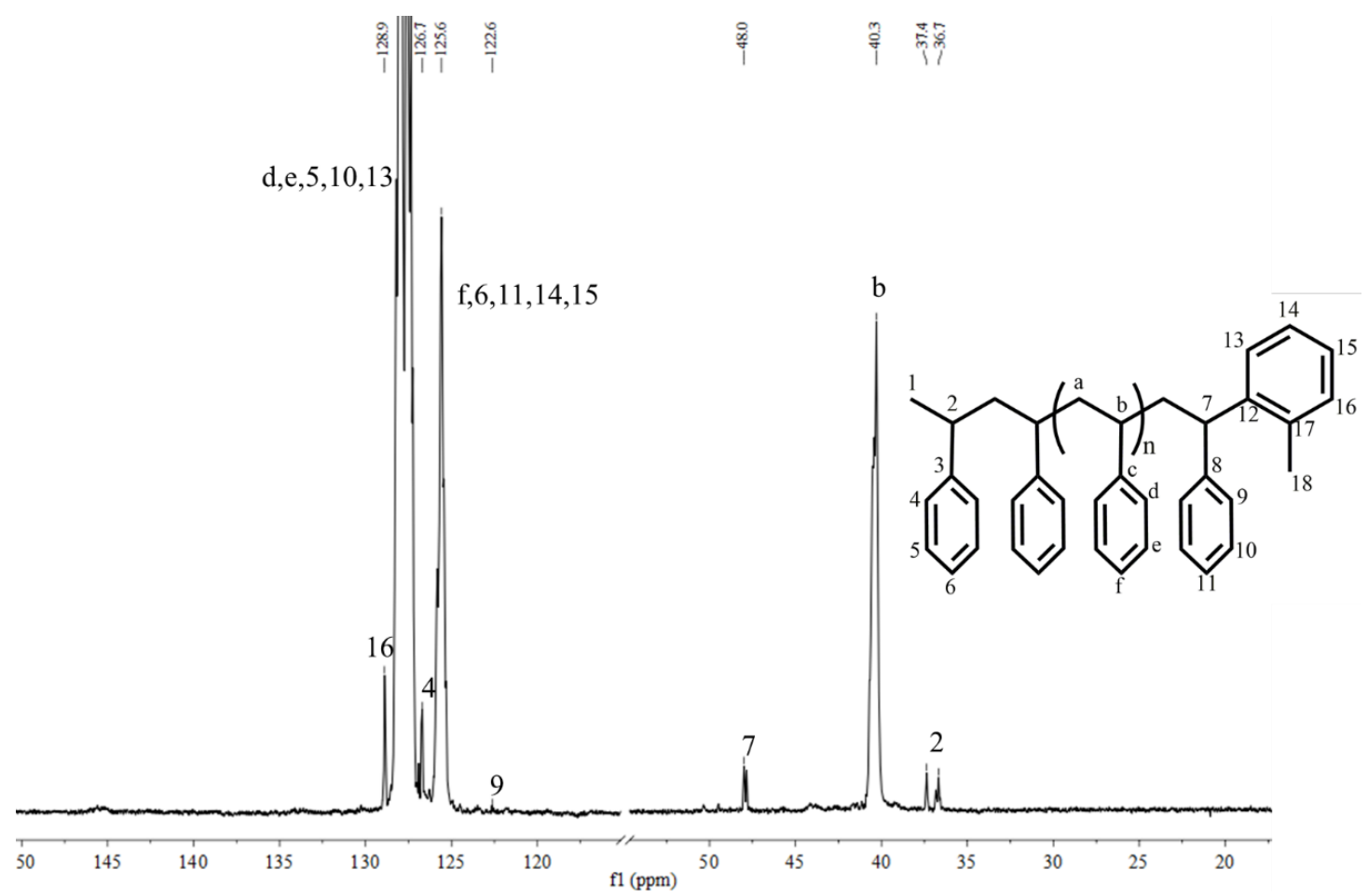

Figure S5. DEPT90 NMR spectrum of polystyrene $\left(M_{\mathrm{n}}=3000, M_{\mathrm{w}} / M_{\mathrm{n}}=1.4\right)$ obtained in toluene. $\left(25^{\circ} \mathrm{C}, \mathrm{CDCl}_{3}\right)$.

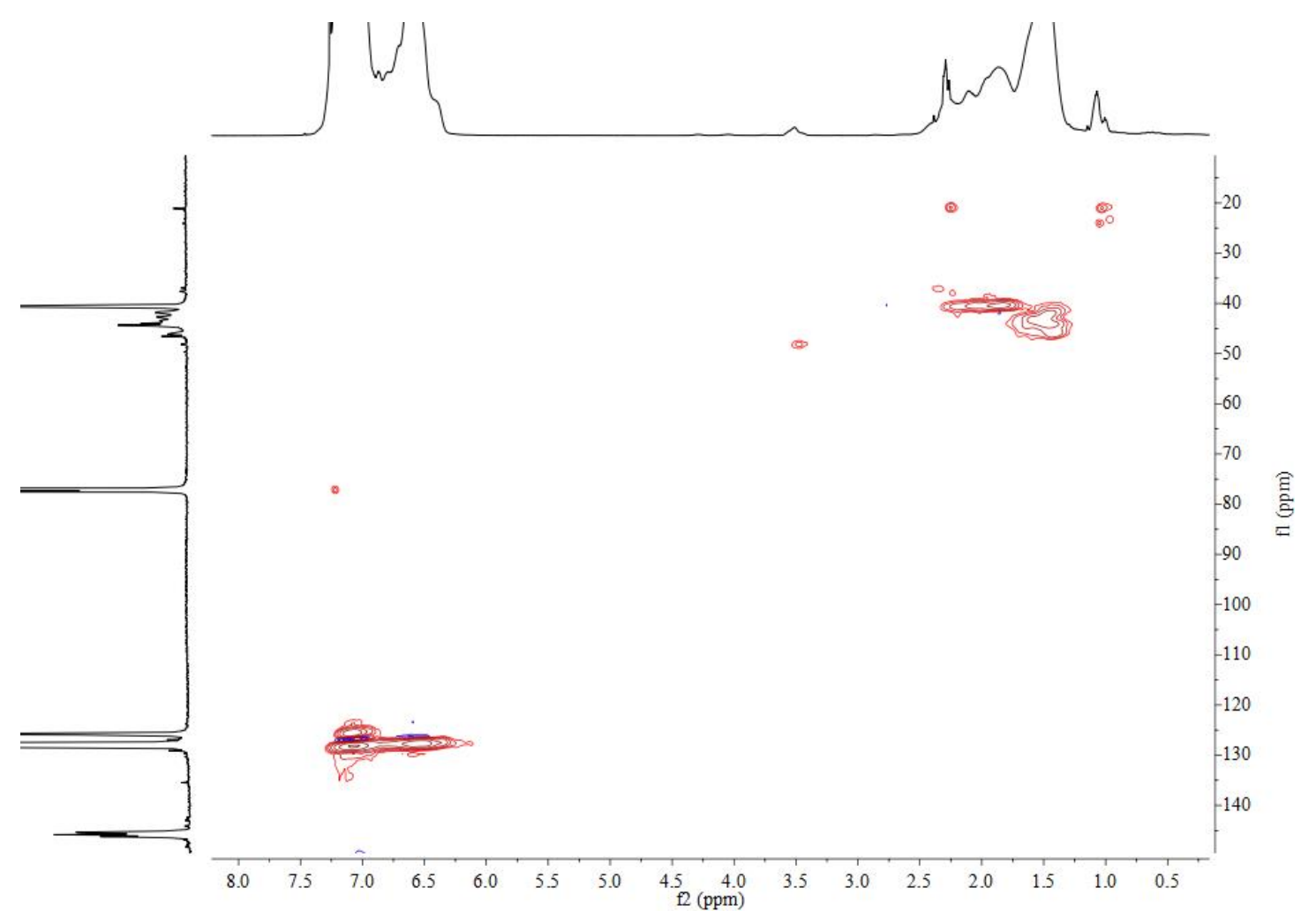

Figure S6. The HSQC NMR spetrum of polystyrene $\left(M_{\mathrm{n}}=3000, M_{\mathrm{w}} / M_{\mathrm{n}}=1.4\right)$ obtained in toluene. $\left(25^{\circ} \mathrm{C}, \mathrm{CDCl}_{3}\right)$. 


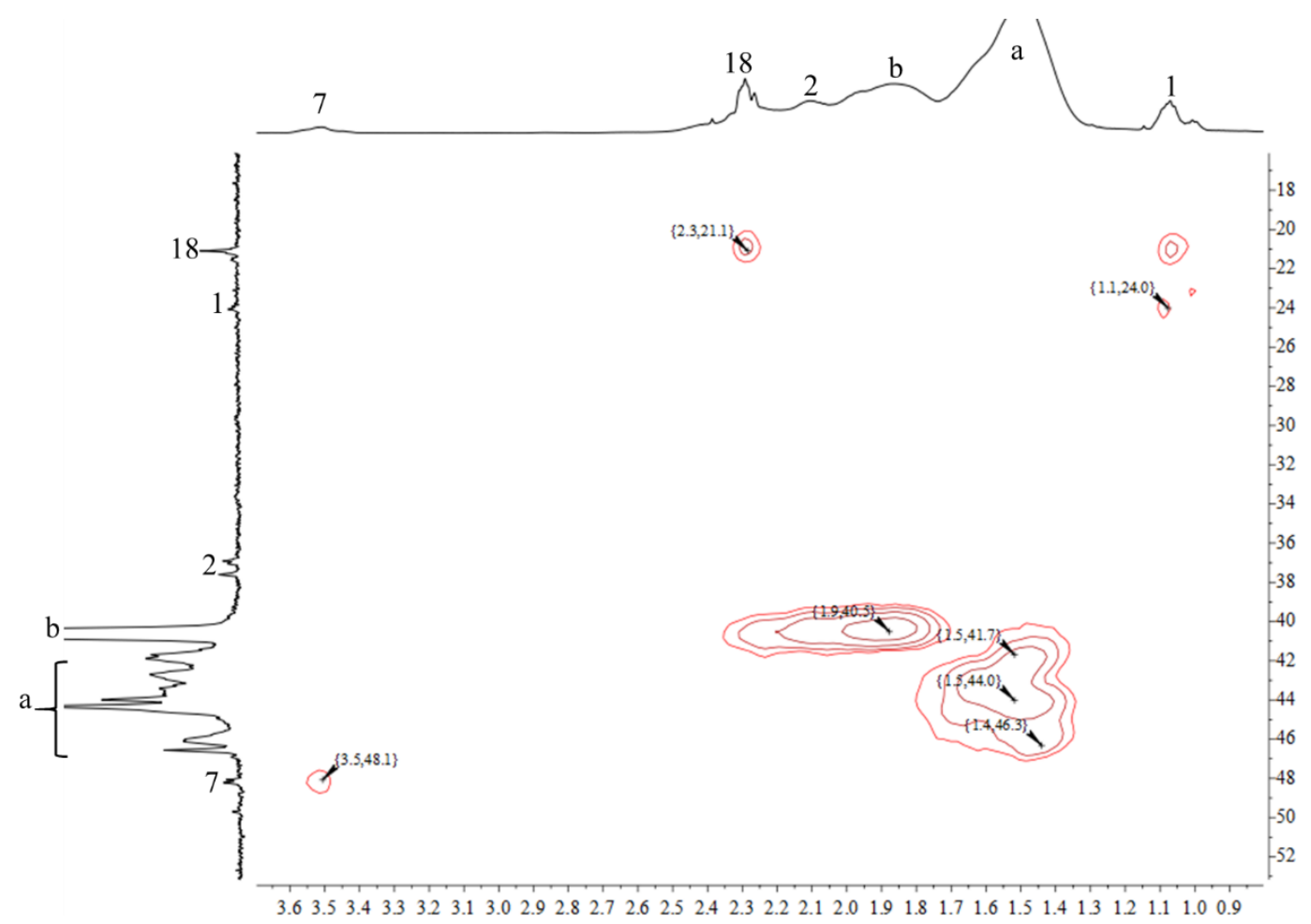

Figure S7. The HSQC NMR spetrum of polystyrene $\left(M_{\mathrm{n}}=3000, M_{\mathrm{w}} / M_{\mathrm{n}}=1.4\right)$ obtained in toluene. (0.8 ppm-3.7ppm in ${ }^{1}$ Hand $16-53$ ppm in ${ }^{13} \mathrm{C}$ ).

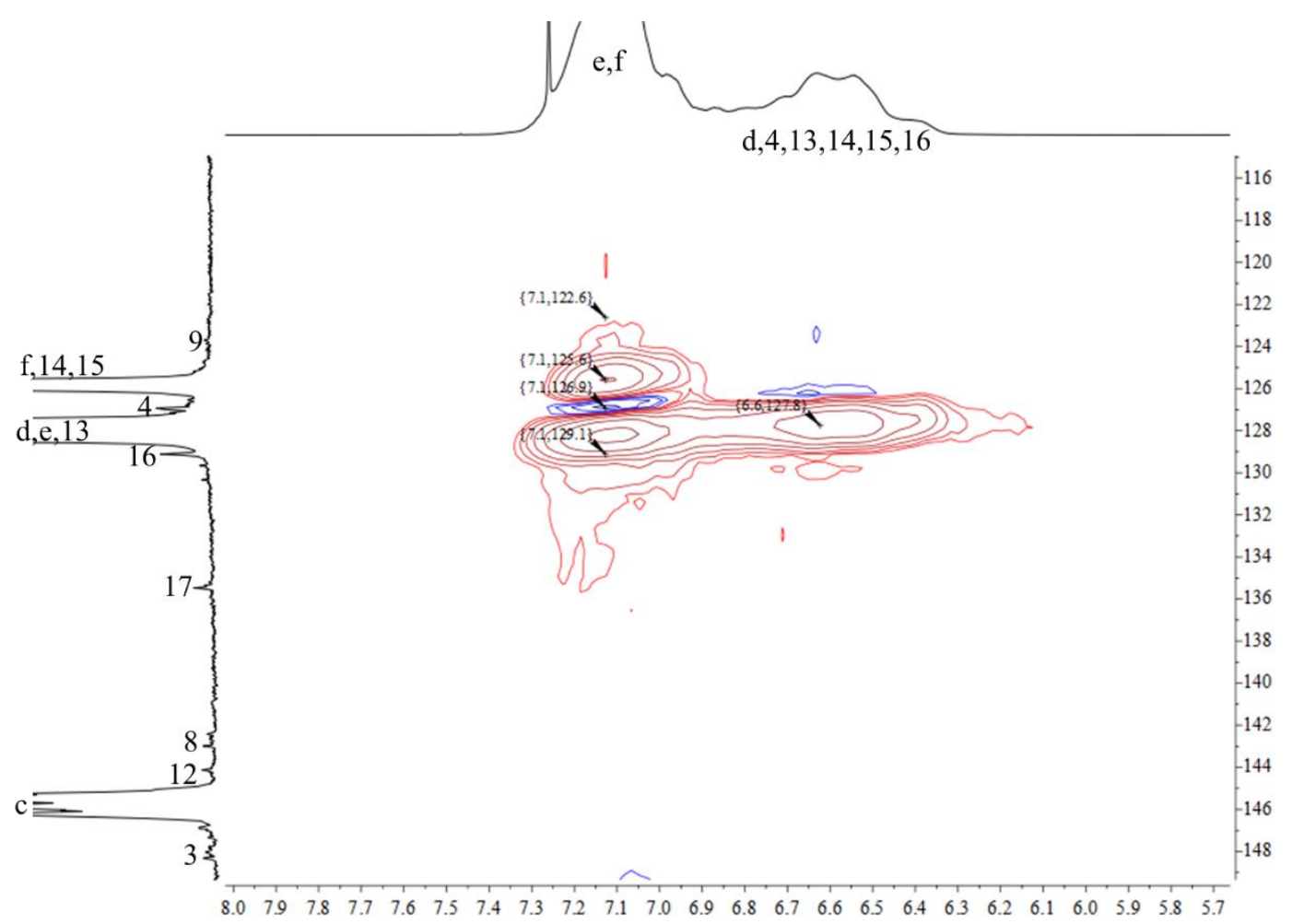

Figure S8. The HSQC NMR spetrum of polystyrene $\left(M_{\mathrm{n}}=3000, M_{\mathrm{w}} / M_{\mathrm{n}}=1.4\right)$ obtained in toluene. (5.7 ppm-8.0 ppm in ${ }^{1} \mathrm{H}$ and $115-149 \mathrm{ppm}$ in ${ }^{13} \mathrm{C}$ ). 


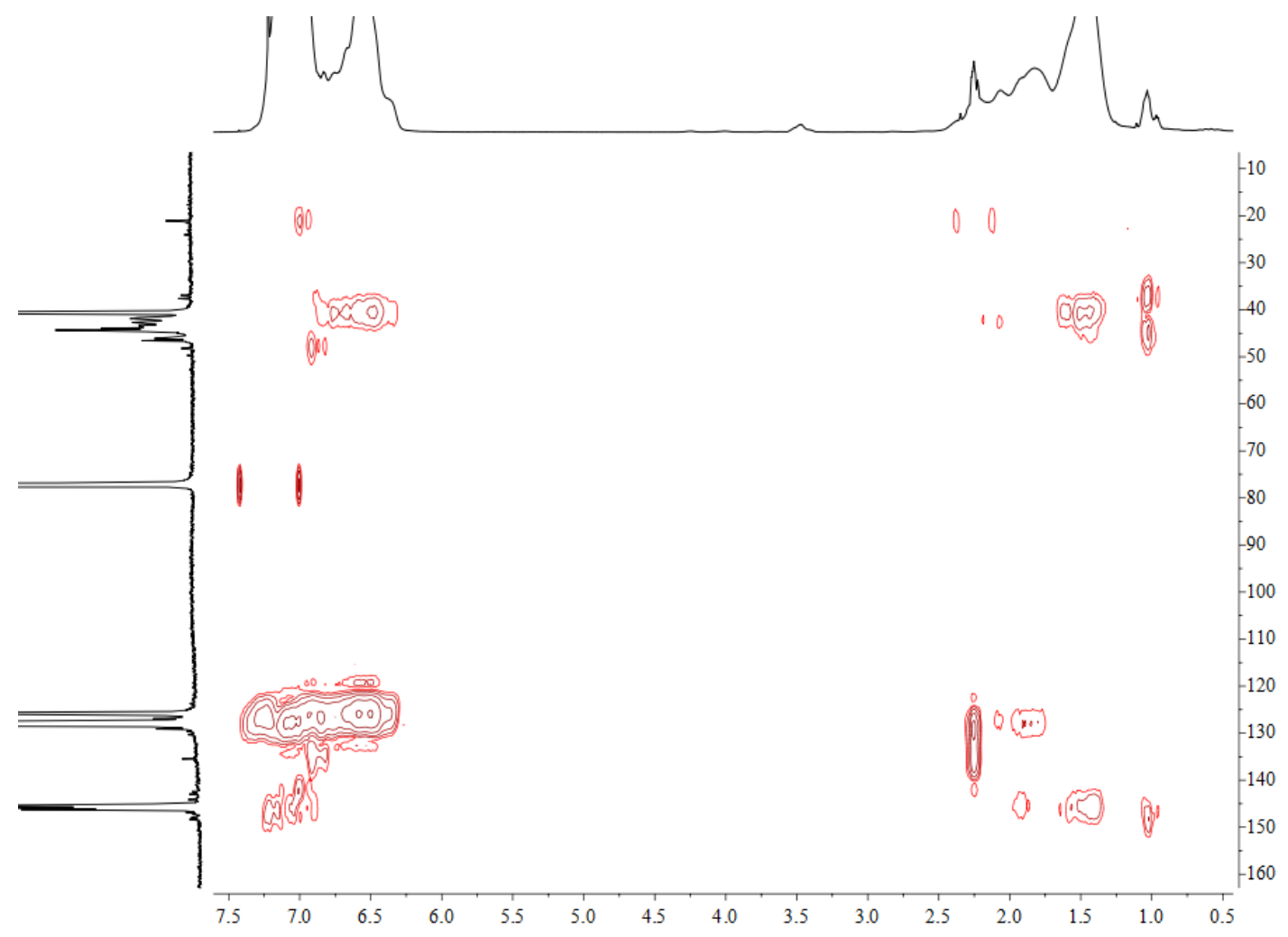

Figure S9. The HMBC NMR spetrum of polystyrene $\left(M_{\mathrm{n}}=3000, M_{\mathrm{w}} / M_{\mathrm{n}}=1.4\right)$ obtained in toluene. $\left(25^{\circ} \mathrm{C}, \mathrm{CDCl}_{3}\right)$.

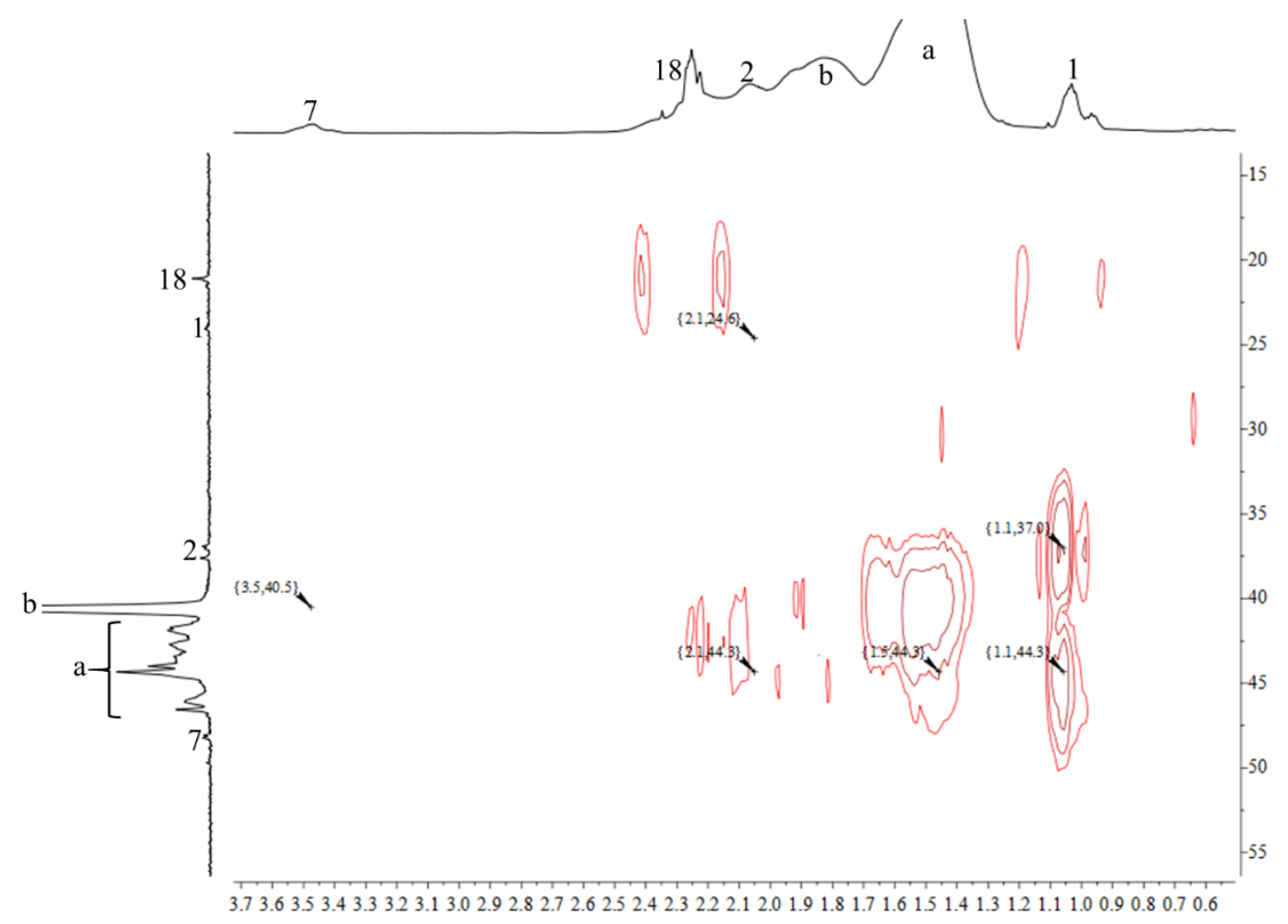

Figure S10. The HMBC NMR spetrum of polystyrene $\left(M_{\mathrm{n}}=3000, M_{\mathrm{w}} / M_{\mathrm{n}}=1.4\right)$ obtained in toluene. (0.5 ppm-3.7 ppm in ${ }^{1} \mathrm{H}$ and $15-55$ ppm in $\left.{ }^{13} \mathrm{C}\right)$. 


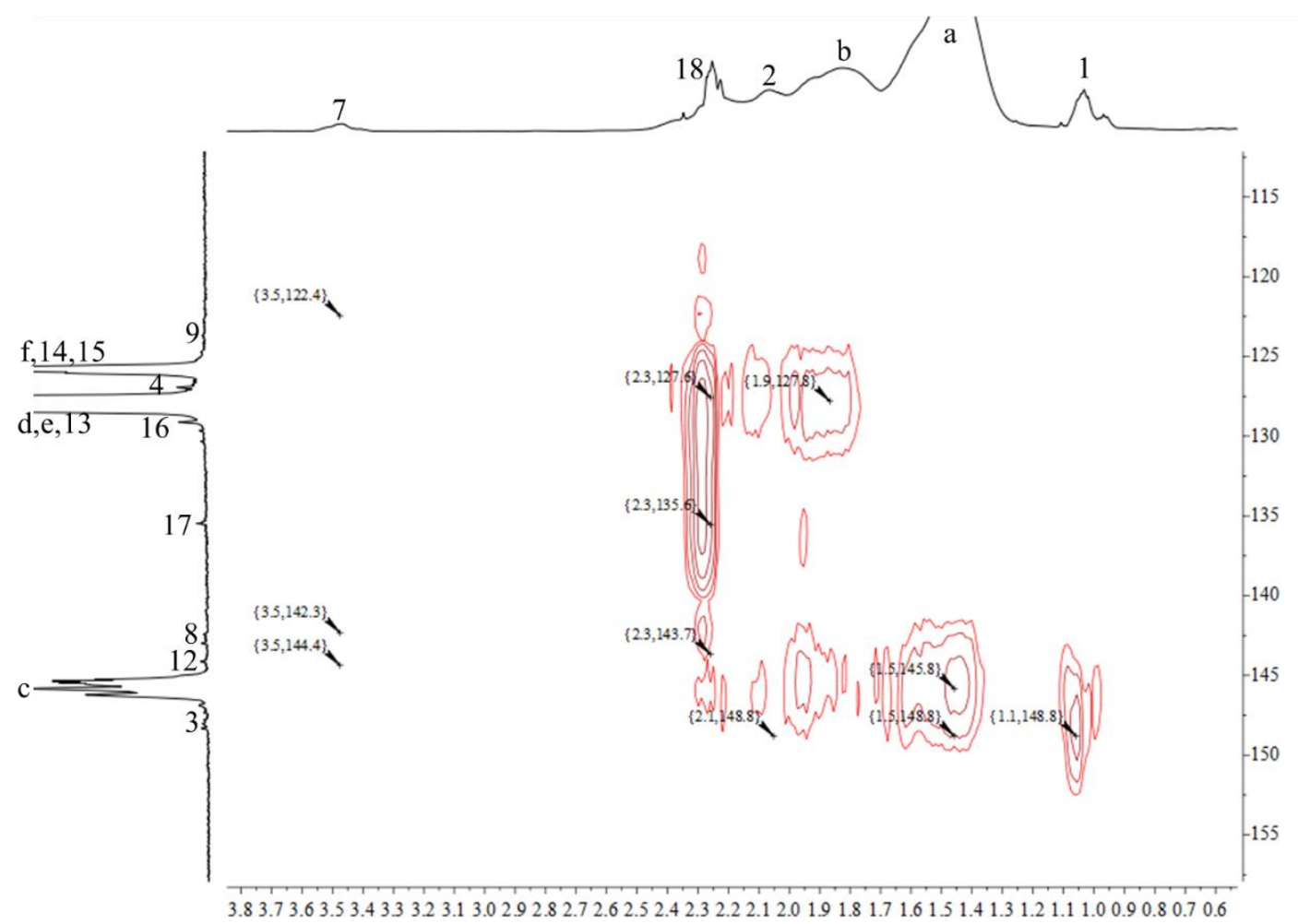

Figure S11. The HMBC NMR spetrum of polystyrene $\left(M_{\mathrm{n}}=3000, M_{\mathrm{w}} / M_{\mathrm{n}}=1.4\right)$ obtained in toluene. (0.5 ppm-3.8 ppm in ${ }^{1} \mathrm{H}$ and $115-155 \mathrm{ppm}$ in $\left.{ }^{13} \mathrm{C}\right)$.

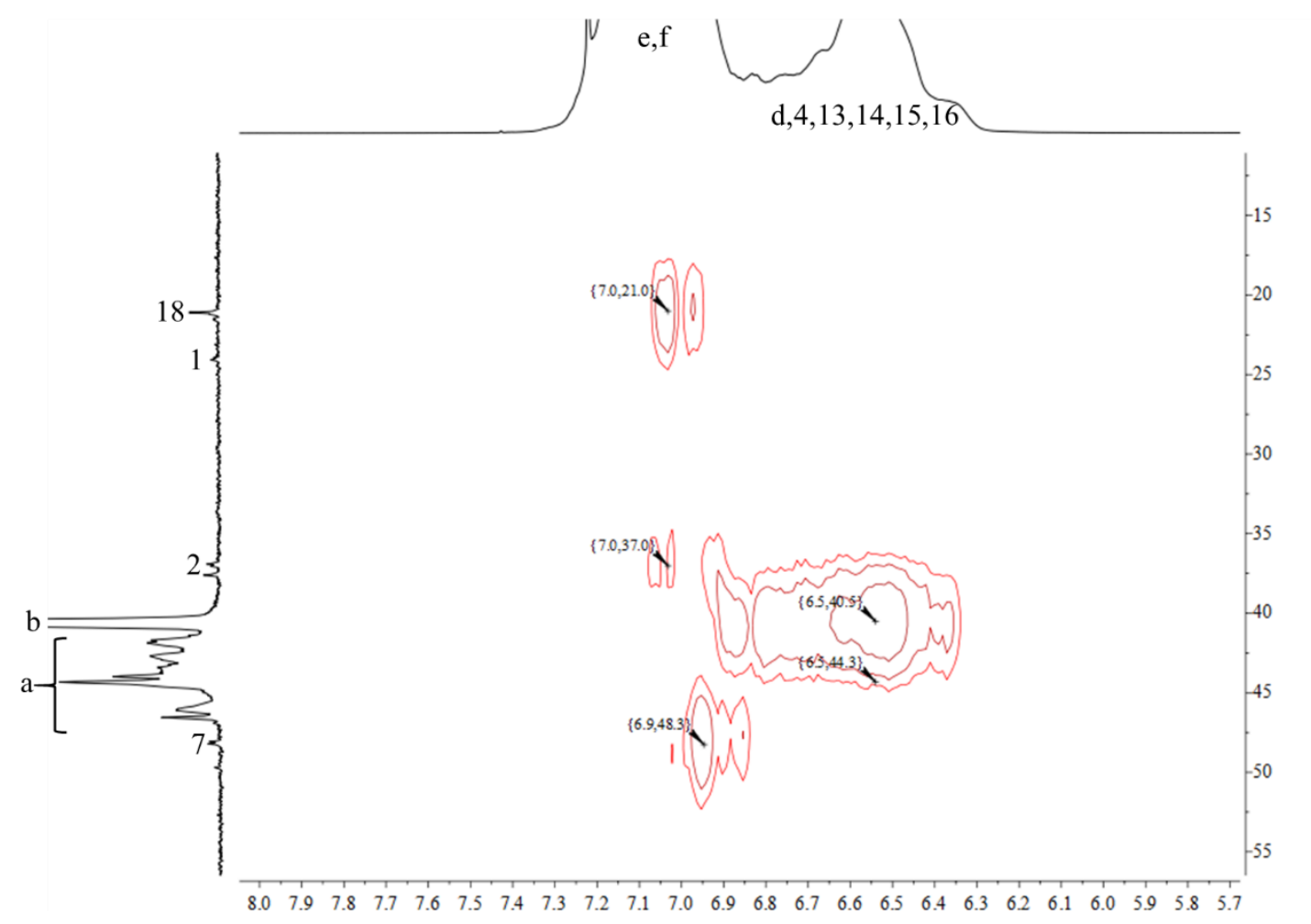

Figure S12. The HMBC NMR spetrum of polystyrene $\left(M_{\mathrm{n}}=3000, M_{\mathrm{w}} / M_{\mathrm{n}}=1.4\right)$ obtained in toluene. (5.7 ppm-8.0 ppm in ${ }^{1} \mathrm{H}$ and $15-55 \mathrm{ppm}$ in $\left.{ }^{13} \mathrm{C}\right)$. 


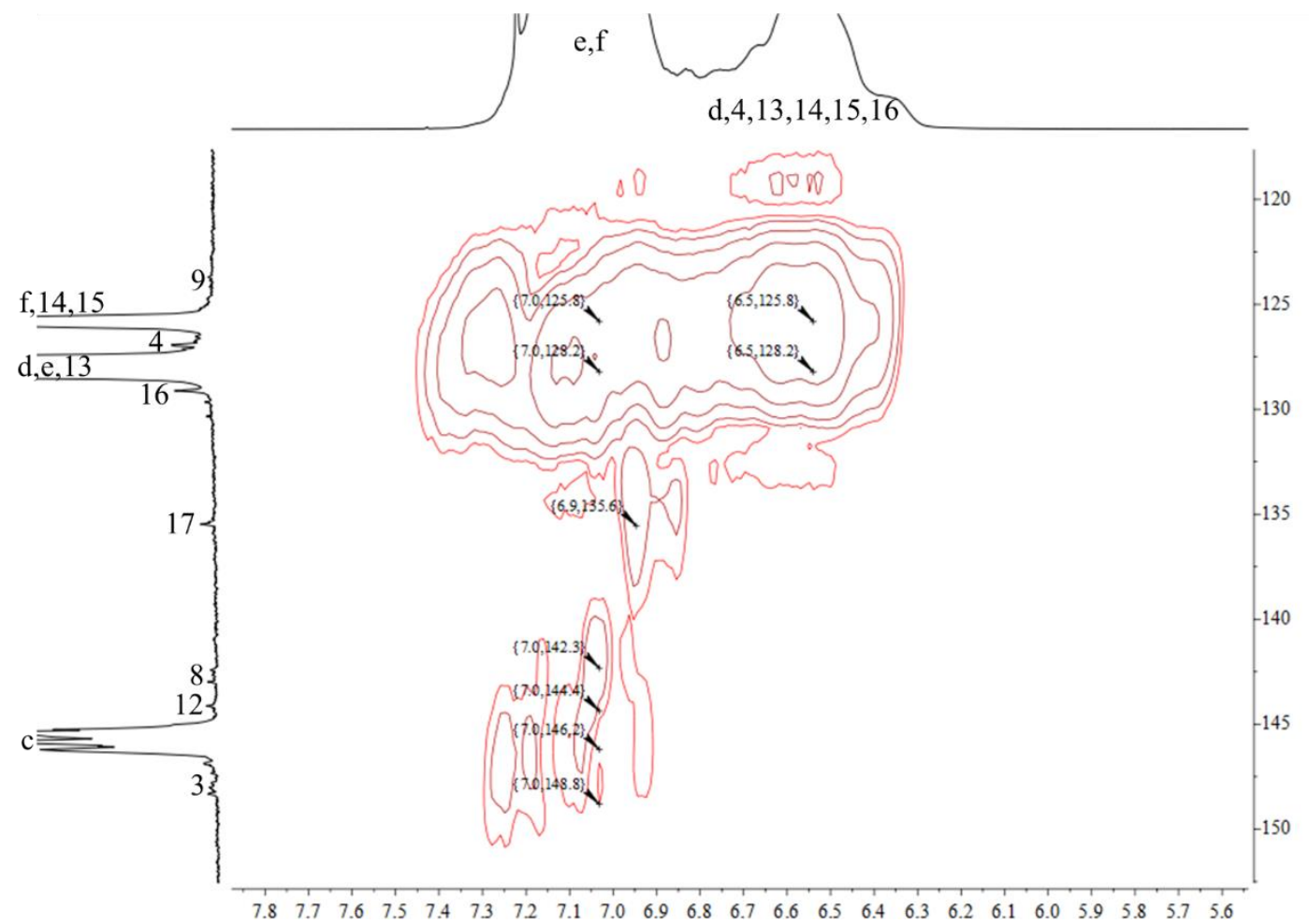

Figure S13. The HMBC NMR spetrum of polystyrene $\left(M_{\mathrm{n}}=3000, M_{\mathrm{w}} / M_{\mathrm{n}}=1.4\right)$ obtained in toluene. (5.6 ppm-7.8 ppm in ${ }^{1} \mathrm{H}$ and $118-152 \mathrm{ppm}$ in ${ }^{13} \mathrm{C}$ ).

Table S1. The Effects of Various Solvent and Catalysts for Styrene Polymerization ${ }^{a}$

\begin{tabular}{cccccccc}
\hline Run & Cat. & solvent & $\begin{array}{c}\text { Conv./ } \\
\%\end{array}$ & $\begin{array}{c}M_{\mathrm{n}} \text { (calcd.) } \\
\times 10^{3}\end{array}$ & $\begin{array}{c}M_{\mathrm{n}}{ }^{b}(\mathrm{exp} .) \\
\times 10^{3}\end{array}$ & $M_{\mathrm{w}} / M_{\mathrm{n}}{ }^{b}$ & Chain $/ \mathrm{Sc}$ \\
\hline 1 & $\mathbf{1}$ & toluene & 57.2 & 23.8 & 3.1 & 1.5 & 7.7 \\
2 & $\mathbf{1}$ & benzene & 54.6 & 22.7 & 8.1 & 1.8 & 2.8 \\
3 & $\mathbf{1}$ & chlorobenzene & 61.3 & 25.5 & 7.0 & 2.1 & 3.6 \\
4 & $\mathbf{1}$ & cyclohexane & 59.1 & 24.6 & 4.2 & 1.7 & 5.9 \\
5 & $\mathbf{2}$ & toluene & 35.7 & 14.8 & 3.3 & 1.7 & 4.5 \\
6 & $\mathbf{3}$ & toluene & 63.8 & 26.5 & 3.1 & 1.8 & 8.5
\end{tabular}

${ }^{a}$ Conditions: $10 \mu \mathrm{mol}$ of catalyst, $10 \mu \mathrm{mol}$ of $\left[\mathrm{Ph}_{3} \mathrm{C}\right]\left[\mathrm{B}\left(\mathrm{C}_{6} \mathrm{~F}_{5}\right)_{4}\right], 10 \mu \mathrm{mol}$ of $\mathrm{Al}^{i} \mathrm{Bu}_{3}, 4 m \mathrm{~mol}$ of styrene, solvent $4 \mathrm{~g}, \mathrm{t}=24 \mathrm{~h}, \mathrm{~T}=20^{\circ} \mathrm{C} .{ }^{b}$ Determined by GPC in THF at $40{ }^{\circ} \mathrm{C}$ against polystyrene standard.

Table S2. Cartesian Coordinates for All of the Calculated Structures 


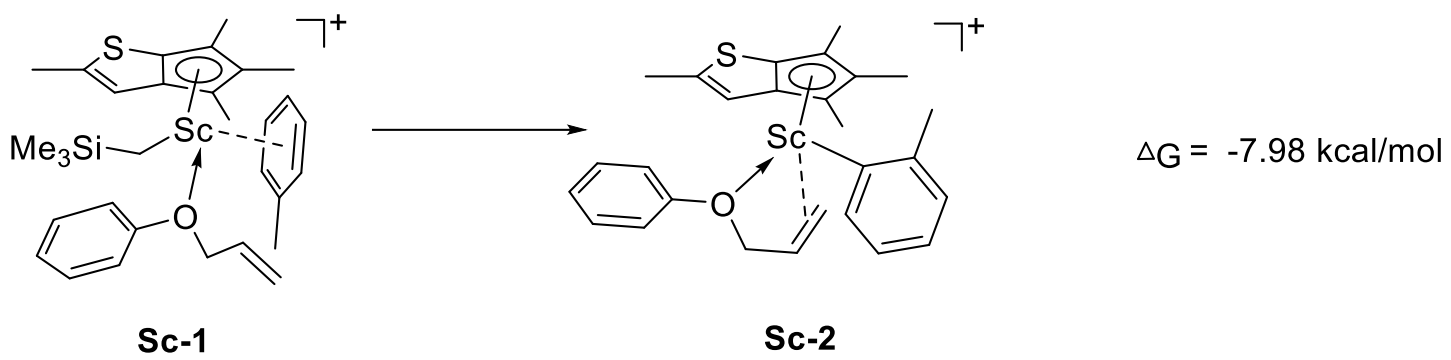

Sc-1

$\mathrm{C}$

C

C

C

C

C

$\mathrm{H}$

$\mathrm{H}$

$\mathrm{H}$

C

$\mathrm{H}$

$\mathrm{H}$

$\mathrm{H}$

C

$\mathrm{H}$

$\mathrm{H}$

$\mathrm{H}$

C

$\mathrm{H}$

C

C

$\mathrm{H}$

$\mathrm{H}$

$\mathrm{H}$

$\mathrm{S}$

Sc

C

C

C

C

C

C

$\mathrm{H}$

$\mathrm{H}$

0.15989200

$-1.12045700$

$-2.75321700$

$-1.01848700$

$-1.42432100$

$-1.99922000$

$-0.65225300$

$-2.32296500$

$-0.95523800$

0.74339000

$-2.58520300$

$-1.01718800$

1.23396900

$-1.85332200$

$-2.15693900$

2.62842500

$-1.96721800$

$-2.71908400$

3.37770300

$-2.16584600$

$-1.94647600$

2.68432000

$-2.79695100$

$-3.43597300$

2.93841100

$-1.06464900$

$-3.25638700$

0.16082600

$-0.41628300$

$-4.08482300$

1.17094900

$-0.21898900$

$-4.45436700$

$-0.33202900$

$-1.04372200$

$-4.83931400$

$-0.38446800$

0.53484000

$-4.06301700$

1.46559100

$-3.63561800$

$-0.21741900$

1.13329200

$-4.63520500$

$-0.52851100$

2.54809300

$-3.59904100$

$-0.36593800$

1.26612000

$-3.55073100$

0.85651200

$-2.44633400$

$-1.24285500$

$-2.08703300$

$-2.94665100$

$-0.61644400$

$-2.81811900$

$-3.13363700$

$-2.02104500$

$-1.20048100$

$-4.61948400$

$-2.14416300$

$-1.04354600$

$-4.95248900$

$-1.83430300$

$-0.04590400$

$-5.12689300$

$-1.51525500$

$-1.78112700$

$-4.95185000$

$-3.17810900$

$-1.19379400$

$-2.06573600$

$-2.98958900$

$-0.16297300$

0.51046200

$-0.17371100$

$-0.45213400$

3.42680900

0.54373800

2.89719900

3.20153900

1.69305800

2.13741600

3.09837500

1.60601300

0.74769900

3.21830400

0.35307000

0.12545400

3.45190500

$-0.79480300$

0.90056100

3.55228300

$-0.71858000$

2.29718700

3.51927400

0.62846000

3.97715600

3.13024600

2.65849900

2.63023400

$\mathrm{H}$

2.96786300

2.49890000

0.14619800

$\mathrm{H}$

3.27536500

0.29229000

$-0.96135000$ 
$\mathrm{H}$

C

$\mathrm{H}$

$\mathrm{H}$

$\mathrm{H}$

$\mathrm{Si}$

$\mathrm{C}$

$\mathrm{H}$

$\mathrm{H}$

$\mathrm{H}$

C

$\mathrm{H}$

$\mathrm{H}$

$\mathrm{H}$

C

$\mathrm{H}$

$\mathrm{H}$

$\mathrm{H}$

C

$\mathrm{H}$

$\mathrm{H}$

C

C

C

C

C

C

$\mathrm{H}$

$\mathrm{H}$

$\mathrm{H}$

$\mathrm{H}$

$\mathrm{H}$

$\mathrm{O}$

C

$\mathrm{H}$

$\mathrm{H}$

C

$\mathrm{H}$

C

$\mathrm{H}$

$\mathrm{H}$

$\begin{array}{ccc}3.60155500 & -1.75212700 & 0.41133100 \\ 3.79404800 & -1.95096100 & 3.13432400 \\ 4.16252900 & -2.78436300 & 2.52891200 \\ 4.52553200 & -1.75696600 & 3.92562600 \\ 2.86811000 & -2.27919100 & 3.62416400 \\ -1.56048200 & -0.27064200 & 2.79785300 \\ -1.16996300 & 1.01672800 & 4.14120400 \\ -0.24753100 & 0.76829600 & 4.68047600 \\ -1.04704300 & 2.02121100 & 3.71659600 \\ -1.97860200 & 1.07100300 & 4.88068300 \\ -1.73461600 & -1.96351800 & 3.63147900 \\ -1.95235800 & -2.7565500 & 2.90761000 \\ -0.81740300 & -2.24004800 & 4.16673500 \\ -2.54961700 & -1.95360800 & 4.36584100 \\ -3.20721300 & 0.21211200 & 1.99649600 \\ -3.17558000 & 1.22836500 & 1.58899200 \\ -3.47135300 & -0.46666000 & 1.17970200 \\ -4.01589600 & 0.17786300 & 2.73748600 \\ -0.10832600 & -0.31050000 & 1.57159800 \\ 0.49122200 & -1.20302500 & 1.83622200 \\ 0.54026100 & 0.55727500 & 1.82893900 \\ -0.91810800 & 2.58209200 & -0.58508700 \\ -2.16111000 & 2.48751000 & -1.20597200 \\ -3.24114300 & 3.17781000 & -0.65067600 \\ -3.06639000 & 3.94691600 & 0.50207900 \\ -1.81125500 & 4.02354800 & 1.11240800 \\ -0.72168200 & 3.34222900 & 0.56587000 \\ -2.28085500 & 1.88797700 & -2.10251900 \\ -4.21722000 & 3.11358700 & -1.12149100 \\ -3.90844700 & 4.48408000 & 0.92724800 \\ -1.67659600 & 4.61774500 & 2.01099200 \\ 0.26258000 & 3.39526700 & 1.02074800 \\ 0.20502200 & 1.92945900 & -1.17027500 \\ 0.79813700 & 2.77014500 & -2.24131800 \\ 0.90184500 & 3.77501900 & -1.82393500 \\ 0.06113000 & 2.79669900 & -3.05077100 \\ 2.11226000 & 2.23891100 & -2.70827900 \\ 2.11148200 & 1.25020600 & -3.15968500 \\ 3.23297400 & 2.96552800 & -2.68871700 \\ 4.16322200 & 2.58944600 & -3.10467400 \\ 3.25805800 & 3.97386400 & -2.28080100\end{array}$

Sc-2

C

1.15985400

$-2.37383100$

$-0.99344200$ 


\begin{tabular}{|c|c|c|c|}
\hline $\mathrm{C}$ & 2.18452600 & -1.50027100 & -0.50144300 \\
\hline $\mathrm{C}$ & 2.00999600 & -0.23144300 & -1.12778900 \\
\hline $\mathrm{C}$ & 0.89468300 & -0.27891900 & -2.01239800 \\
\hline $\mathrm{C}$ & 0.40400200 & -1.63261000 & -1.95682700 \\
\hline $\mathrm{C}$ & -0.65431000 & -2.21201000 & -2.85528400 \\
\hline $\mathrm{H}$ & -1.34469200 & -1.44660000 & -3.21914200 \\
\hline $\mathrm{H}$ & -0.18929600 & -2.67806200 & -3.73420100 \\
\hline $\mathrm{H}$ & -1.24631300 & -2.97876400 & -2.34781100 \\
\hline $\mathrm{C}$ & 1.02945400 & -3.84519900 & -0.69735300 \\
\hline $\mathrm{H}$ & -0.01191700 & -4.18066600 & -0.72721100 \\
\hline $\mathrm{H}$ & 1.58451600 & -4.43799000 & -1.43595200 \\
\hline $\mathrm{H}$ & 1.43610600 & -4.09791800 & 0.28819800 \\
\hline $\mathrm{C}$ & 0.44582200 & 0.81008200 & -2.94918100 \\
\hline $\mathrm{H}$ & 1.01526300 & 0.76776100 & -3.88699200 \\
\hline $\mathrm{H}$ & -0.61344100 & 0.71934300 & -3.20702800 \\
\hline $\mathrm{H}$ & 0.60000100 & 1.80569100 & -2.51964300 \\
\hline $\mathrm{C}$ & 3.33204000 & -1.52570000 & 0.37366500 \\
\hline $\mathrm{H}$ & 3.66672700 & -2.39949200 & 0.92342300 \\
\hline $\mathrm{C}$ & 4.01722300 & -0.34461700 & 0.39203000 \\
\hline $\mathrm{C}$ & 5.28602900 & -0.01958200 & 1.12289000 \\
\hline $\mathrm{H}$ & 5.59781300 & -0.87547600 & 1.72880700 \\
\hline $\mathrm{H}$ & 6.09943800 & 0.21327400 & 0.42544900 \\
\hline $\mathrm{H}$ & 5.17137500 & 0.84506500 & 1.78796400 \\
\hline $\mathrm{S}$ & 3.26712800 & 0.89540000 & -0.64594400 \\
\hline $\mathrm{Sc}$ & -0.02106700 & -0.59249800 & 0.21138200 \\
\hline $\mathrm{C}$ & -4.38879300 & -1.78365500 & 0.27962400 \\
\hline $\mathrm{C}$ & -4.11500400 & -2.74065300 & 1.26668400 \\
\hline $\mathrm{C}$ & -2.80750000 & -2.95838400 & 1.68830700 \\
\hline $\mathrm{C}$ & -1.79637200 & -2.18291000 & 1.10335100 \\
\hline $\mathrm{C}$ & -2.03958400 & -1.20939200 & 0.12153200 \\
\hline $\mathrm{C}$ & -3.37391500 & -1.02265000 & -0.31024000 \\
\hline $\mathrm{H}$ & -5.41872300 & -1.63294600 & -0.03629300 \\
\hline $\mathrm{H}$ & -4.92859800 & -3.31739700 & 1.69734100 \\
\hline $\mathrm{H}$ & -2.57960600 & -3.71058800 & 2.43819800 \\
\hline $\mathrm{H}$ & -0.76392400 & -2.41044600 & 1.43833800 \\
\hline $\mathrm{C}$ & -3.71001700 & -0.03507000 & -1.40541100 \\
\hline $\mathrm{H}$ & -3.17252000 & 0.91088400 & -1.27725500 \\
\hline $\mathrm{H}$ & -3.43156300 & -0.43495200 & -2.38925300 \\
\hline $\mathrm{H}$ & -4.78217100 & 0.18361200 & -1.43712900 \\
\hline $\mathrm{C}$ & -0.66472600 & 2.65852500 & 0.49659700 \\
\hline $\mathrm{C}$ & 0.17117500 & 3.59753900 & -0.10265000 \\
\hline $\mathrm{C}$ & -0.40067100 & 4.76364300 & -0.61712800 \\
\hline $\mathrm{C}$ & -1.77893800 & 4.97341600 & -0.52127800 \\
\hline $\mathrm{C}$ & -2.59513200 & 4.01850400 & 0.08971300 \\
\hline
\end{tabular}




$\begin{array}{lrrr}\mathrm{C} & -2.03942200 & 2.84627200 & 0.60971800 \\ \mathrm{H} & 1.23988000 & 3.41620500 & -0.16493600 \\ \mathrm{H} & 0.23318200 & 5.50671600 & -1.09124800 \\ \mathrm{H} & -2.21673200 & 5.88229400 & -0.92220400 \\ \mathrm{H} & -3.66566500 & 4.18262100 & 0.16523900 \\ \mathrm{H} & -2.65722200 & 2.09142200 & 1.08652100 \\ \mathrm{O} & -0.09206500 & 1.46497100 & 1.01054000 \\ \mathrm{C} & 0.36632600 & 1.53457700 & 2.40319600 \\ \mathrm{H} & -0.51281100 & 1.63638100 & 3.04638300 \\ \mathrm{H} & 1.00351000 & 2.41751800 & 2.50253500 \\ \mathrm{C} & 1.12248100 & 0.26066600 & 2.66075400 \\ \mathrm{H} & 2.14590700 & 0.21131800 & 2.29212200 \\ \mathrm{C} & 0.59056200 & -0.78896800 & 3.30725000 \\ \mathrm{H} & 1.16599800 & -1.69443700 & 3.48376100 \\ \mathrm{H} & -0.41836800 & -0.76224300 & 3.71443900\end{array}$

$\mathrm{SiMe}_{4}$

$\mathrm{Si}$

C

$\mathrm{H}$

$\mathrm{H}$

$\mathrm{H}$

C

$\mathrm{H}$

$\mathrm{H}$

$\mathrm{H}$

C

$\mathrm{H}$

$\mathrm{H}$

$\mathrm{H}$

C

$\mathrm{H}$

$\mathrm{H}$

0.00026900

0.00027300

0.00002300

1.83853700

0.10851200

$-0.45111100$

2.44615600

0.40253500

0.41359500

2.21977500

$-0.85568400$

$-0.80930100$

2.01337600

0.84689700

$-1.24325100$

$-0.60170400$

1.69077900

0.61140600

$-1.66432100$

1.66304400

0.88234600

$-0.04403800$

2.01785100

1.49764000

$-0.47806000$

2.46203600

$-0.15880800$

$-0.99743200$

$-0.51015900$

$-1.52918800$

$-0.67210500$

$-1.48543900$

$-1.91165600$

$-2.06784000$

$-0.58596500$

$-1.30139700$

$-0.88427400$

0.21711300

$-2.34260100$

$-0.23964600$

$-1.28937900$

1.36885300

$-1.29522200$

$-1.37877000$

1.65364700

0.32403100

$-1.02409700$

2.27168400

0.10021900

$-2.28186500$

1.04802000

$\mathrm{MePh}$

C

C

C

$-0.19441400$

1.20232600

$-0.00894300$

1.20111300

1.20545400

0.00218000

1.90500900

0.00014100

0.00845200

1.20123000

$-1.20538900$

0.00208500

$-0.19416200$

$-1.20244500$

$-0.00883600$

C

$-0.91380800$

$-0.00005500$

$-0.01121200$

$\mathrm{H}$

$-0.73465400$

2.14679400

$-0.01780800$

$\mathrm{H}$

1.73838200

2.15054400

0.00155100 


$\begin{array}{lrrc}\mathrm{H} & 2.99177300 & 0.00002300 & 0.01396600 \\ \mathrm{H} & 1.73890700 & -2.15024800 & 0.00127500 \\ \mathrm{H} & -0.73453100 & -2.14681200 & -0.01756900 \\ \mathrm{C} & -2.42539900 & -0.00010200 & 0.00928400 \\ \mathrm{H} & -2.83325800 & -0.89353400 & -0.47562500 \\ \mathrm{H} & -2.81012200 & 0.01524900 & 1.03799200 \\ \mathrm{H} & -2.83391000 & 0.87839900 & -0.50184900\end{array}$

